\title{
Tratamiento de la osteonecrosis de los maxilares asociada a medicamentos: Enfoque médico-quirúrgico de estadios II y III
}

\section{Treatment of medication related osteonecrosis of the jaws: Stage II and III medical and surgical approach}

\author{
Hernán Ramírez S. ${ }^{1}$, Francisco Rojas C. ${ }^{1}$, Cristián Teuber L. ${ }^{1}$, \\ Camila Foncea R. ${ }^{1}$, Ignacio Goñi E. ${ }^{1}$, Álex Vargas D. ${ }^{1}$
}

\begin{abstract}
Resumen
Introducción: La osteonecrosis de los maxilares asociada a medicamentos (OMAM) se define como la presencia de hueso necrótico expuesto de los maxilares en pacientes con historia de tratamiento farmacológico antirresortivo o antiangiogénico. Se describen diferentes estadios se severidad, con tratamiento conservador para estadios 0 y I, y tratamiento médico-quirúrgico para II-III. Objetivo: Describir los factores desencadenantes, opciones de tratamiento médico-quirúrgico y resultados en pacientes con OMAM estadios II-III. Material y Método: Estudio retrospectivo, descriptivo, de pacientes diagnosticados con OMAM estadios II y III que requirieron manejo médico-quirúrgico en la Red de Salud UC-Christus entre los años 2007 y 2018. Resultados: Todos los pacientes presentaron historia de tratamiento con bifosfonatos intravenosos. La mayoría de los registros de seguimiento de pacientes estuvo disponible para su análisis. El tratamiento consistió en aseo quirúrgico, decorticación y secuestrectomía. Se reportó disminución de la sintomatología con resolución parcial en la mitad de los casos y cierre completo de la exposición ósea en los restantes. Conclusión: Sugerimos que el tratamiento médico-quirúrgico en pacientes con OMAM en etapas II y III es efectivo en términos de disminución de sintomatología y control de infección. Sin embargo, es necesario realizar nuevos estudios prospectivos, con mayor cantidad de pacientes y tiempo de seguimiento.
\end{abstract}

Palabras clave: Osteonecrosis de los maxilares, bifosfonatos, antiangiogénicos, ácido zoledrónico.

\begin{abstract}
Introduction: Medication-associated osteonecrosis of the jaws (MRONJ) is defined as the presence of exposed necrotic bone of the jaws in patients with a history of antiresorptive or antiangiogenic drug treatment. Different stages of severity are described, with conservative treatment for stages 0 and I, and medical-surgical treatment for II-III. Aim: To describe the triggers, medical-surgical treatment options and outcomes in patients with stage II-III MRONJ. Material and Method: Retrospective, descriptive study of patients diagnosed with MRONJ stages II and III that required medical-surgical management in the UC-Christus Health Network between 2007 and 2018. Results: All patients had a history of treatment with intravenous bisphosphonates. Most of the patient follow-up records were available for analysis. Treatment consisted of surgical grooming, decortication, and sequestrectomy. A decrease in symptoms was reported with partial resolution in half of the cases, and complete closure of bone exposure in the remainder. Conclusion: We suggest that medical-surgical treatment in patients with MRONJ in stages II and III is effective in terms of reducing symptoms and controlling infection. However, it is necessary to carry out new prospective studies, with a greater number of patients and follow-up time.
\end{abstract}

Keywords: Osteonecrosis of the Jaw, bisphosphonates, antiangiogenics, zoledronic acid.
'Departamento de Cirugía Oncológica y Maxilofacial, División de Cirugía, Facultad de Medicina Pontificia Universidad Católica de Chile. Santiago, Chile.

Los autores declaran no tener conflictos de interés.

Recibido el 19 de octubre de 2020. Aceptado el 17 de enero de 2021.

Correspondencia: Alex Vargas D. Av. Diagonal Paraguay 362, $3^{\text {er }}$ Piso.

Departamento de Cirugía Oncológica y Maxilofacial División de Cirugía, Facultad de Medicina, Pontificia Universidad Católica de Chile. Santiago, Chile. Email: avargasi@uc.cl 


\section{Introducción}

La osteonecrosis de los maxilares asociada a medicamentos (OMAM) es definida por la American Association of Oral and Maxillofacial Surgeons (AAOMS) como la presencia de hueso necrótico expuesto o de una fístula maxilofacial con una evolución clínica de al menos 8 semanas. Se observa en pacientes que han sido tratados con fármacos antirresortivos o antiangiogénicos, que no hayan recibido radioterapia ni presenten metástasis óseas maxilofaciales evidentes ${ }^{1-3}$. Fue descrita inicialmente por Marx el año 2003 y desde esa fecha los reportes de esta patología han tenido un crecimiento exponencial ${ }^{1}$.

La OMAM ha sido asociada con mayor frecuencia al uso de bifosfonatos administrados por vía intravenosa (BFIV), indicados habitualmente para el tratamiento de la osteoporosis severa, metástasis óseas e hipercalcemia maligna. Su prevalencia varía entre un $0,94 \%$ y un $13 \%$ en diferentes grupos de población con diferentes regímenes de drogas, siendo el principal factor desencadenante la exodoncia u otro procedimiento odontológico invasivo ${ }^{4-8}$. Si bien la frecuencia de esta patología es en general baja, la OMAM suele afectar negativamente la calidad de vida de los pacientes y se asocia a complicaciones severas, a pesar de un tratamiento adecuado.

Actualmente no existe un consenso respecto al tratamiento de la OMAM. Sin embargo, los principales objetivos terapéuticos son manejo de dolor e infección, minimizar la progresión de necrosis óseas y establecer un ambiente adecuado para la cicatrización del tejido óseo ${ }^{1,2}$. En pacientes con pequeñas áreas necróticas o estadios iniciales de la enfermedad, el tratamiento suele ser conservador orientado a disminuir los síntomas y mejorar los factores locales, principalmente mediante la administración de antimicrobianos (tópicos y sistémicos) y analgésicos ${ }^{2}$. Pacientes con extensas áreas de osteonecrosis o mala respuesta al manejo conservador, requieren intervenciones quirúrgicas con el objetivo de eliminar secuestros óseos y realizar un adecuado aseo quirúrgico $^{2-9}$. Además del tratamiento médico quirúrgico, existen terapias complementarias como el uso de concentrados plaquetarios, administración vía oral de pentoxifilina más tocoferol (PENT-E) y terapia de oxígeno hiperbárico $^{10}$.

\section{Objetivo}

El objetivo de este trabajo es describir los posibles factores desencadenantes, opciones de tratamiento y evolución luego del tratamiento médico-quirúrgico de pacientes con OMAM en estadios avanzados II-III.

\section{Material y Método}

Se diseñó un estudio retrospectivo, descriptivo, de todos los pacientes diagnosticados con OMAM en estadios II y III que consultaron en la Red de Salud UC-Christus (Santiago, Chile) entre los años 2007 y 2018, y que requirieron manejo médico-quirúrgico como parte de su tratamiento. Ingresaron al estudio 9 pacientes, de los cuales se registraron datos de: género, edad, diagnóstico primario, terapia antirresortiva, tiempo de duración de la terapia, estadio inicial, ubicación anatómica de la lesión, evento desencadenante, tratamiento médico y quirúrgico realizado, tiempo de seguimiento, resultados de cultivos aerobio y anaerobio, análisis anatomopatológico y estadio de la lesión al último control. La información fue obtenida desde bases de datos y fichas clínicas de los pacientes evaluados en el período señalado.

Para definir el estadio de la OMAM fue utilizada la clasificación de la American Association of Oral and Maxillofacial Surgeons $(\mathrm{AAOMS})^{2}$ :

- Estadio 0: Sin evidencia clínica de hueso necrótico expuesto, en presencia de hallazgos clínicos no específicos, cambios radiográficos y sintomatología.

- Estadio I: Presencia de hueso necrótico expuesto o fístula que conecta con el hueso, en pacientes asintomáticos y sin signos de infección.

- Estadio II: Presencia de hueso necrótico expuesto o fístula que conecta con el hueso, asociado a infección, dolor y eritema de la región del hueso expuesto, con o sin presencia de exudado purulento. 
- Estadio III: Presencia de hueso necrótico expuesto o fístula que conecta con el hueso, asociado a infección, dolor y eritema de la región del hueso expuesto y uno o más de los siguientes signos: Hueso necrótico expuesto extendido más allá del hueso alveolar, fractura patológica, fístula extraoral, comunicación oroantral.

Los criterios de inclusión se remitieron a los pacientes que presentaran historia de tratamiento con fármacos antirresortivos previo o durante la aparición de la patología y que hayan requerido tratamiento médico-quirúrgico. Los pacientes con antecedentes de radioterapia de cabeza y cuello o metástasis óseas en los maxilares fueron excluidos del estudio. El trabajo fue aprobado por el Comité de Ética de la Escuela de Medicina de la Pontificia Universidad Católica de Chile. El ingreso y análisis de datos se realizó a través del programa Epi Info (Versión 7.0, CDC, Atlanta, USA).

\section{Resultados}

El registro de seguimiento completo estuvo disponible en 6 casos. En 3 pacientes el seguimiento fue igual o menor a 3 meses. En estos casos se registró una disminución de los síntomas y del tejido óseo expuesto (estadio I). En 3 pacientes se observó un seguimiento igual o mayor a 12 meses, en los cuales se evidenció una disminución considerable de los síntomas, con cobertura total de la exposición ósea (estadio 0). La Tabla 1 muestra un resumen de los hallazgos clínicos de los pacientes, mientras que la Tabla 2 resume el tratamiento realizado en cada caso y la progresión de la osteonecrosis al último control, para el cual los pacientes se mantenían en tratamiento con bifosfonatos por su patología de base.

Todos los casos corresponden a pacientes de género femenino, con una edad promedio de 62,1 años al momento del diagnóstico. Todos tenían historia de tratamiento con BFIV, en un

\begin{tabular}{|c|c|c|c|c|c|c|c|c|}
\hline Caso & Género & Edad & $\begin{array}{c}\text { Diagnóstico } \\
\text { primario }\end{array}$ & $\begin{array}{c}\text { Fármaco } \\
\text { asociado a } \\
\text { OMAM }\end{array}$ & $\begin{array}{c}\text { Duración de } \\
\text { fármaco asociado } \\
\text { a OMAM }\end{array}$ & $\begin{array}{c}\text { Estadio } \\
\text { inicial }\end{array}$ & $\begin{array}{l}\text { Ubicación } \\
\text { anatómica }\end{array}$ & $\begin{array}{c}\text { Evento } \\
\text { precipitante }\end{array}$ \\
\hline$N^{0} 1$ & Femenino & 53 & $\begin{array}{l}\text { Mieloma } \\
\text { múltiple }\end{array}$ & $\begin{array}{c}\text { Ácido } \\
\text { zolendrónico }\end{array}$ & 18 meses & 2 & $\begin{array}{l}\text { Maxilar } \\
\text { derecho }\end{array}$ & $\begin{array}{c}\text { Compresión } \\
\text { protésica }\end{array}$ \\
\hline$N^{\circ} 2$ & Femenino & 37 & $\begin{array}{l}\text { Mieloma } \\
\text { múltiple }\end{array}$ & $\begin{array}{l}\text { Rituximab } \\
\text { Ácido } \\
\text { zolendrónico }\end{array}$ & $\begin{array}{l}12 \text { meses } \\
1 \text { mes }\end{array}$ & 3 & $\begin{array}{l}\text { Maxilar } \\
\text { izquierdo }\end{array}$ & $\begin{array}{l}\text { Extracción } \\
\text { dental }\end{array}$ \\
\hline$N^{0} 3$ & Femenino & 61 & $\begin{array}{c}\text { Osteoporosis } \\
\text { severa }\end{array}$ & $\begin{array}{c}\text { Ácido } \\
\text { zolendrónico }\end{array}$ & 24 meses & 2 & $\begin{array}{l}\text { Mandibular } \\
\text { derecho }\end{array}$ & $\begin{array}{l}\text { Extracción } \\
\text { dental }\end{array}$ \\
\hline$N^{\circ} 4$ & Femenino & 73 & $\begin{array}{c}\text { Osteoporosis } \\
\text { severa }\end{array}$ & Alendronato & 120 meses & 2 & $\begin{array}{l}\text { Mandibular } \\
\text { derecho }\end{array}$ & $\begin{array}{l}\text { Extracción } \\
\text { dental }\end{array}$ \\
\hline$N^{\circ} 5$ & Femenino & 75 & $\begin{array}{l}\text { Cáncer de } \\
\text { mama }\end{array}$ & $\begin{array}{c}\text { Ácido } \\
\text { zolendrónico }\end{array}$ & 72 meses & 2 & $\begin{array}{l}\text { Mandibular } \\
\text { derecho }\end{array}$ & $\begin{array}{l}\text { Extracción } \\
\text { dental }\end{array}$ \\
\hline$N^{\circ} 6$ & Femenino & 65 & $\begin{array}{l}\text { Cáncer de } \\
\text { mama }\end{array}$ & $\begin{array}{c}\text { Ácido } \\
\text { zolendrónico }\end{array}$ & 28 meses & 2 & $\begin{array}{l}\text { Mandibular } \\
\text { derecho }\end{array}$ & $\begin{array}{l}\text { Extracción } \\
\text { dental }\end{array}$ \\
\hline$N^{0} 7$ & Femenino & 66 & $\begin{array}{l}\text { Cáncer de } \\
\text { mama }\end{array}$ & $\begin{array}{c}\text { Ácido } \\
\text { zolendrónico }\end{array}$ & 6 meses & 2 & $\begin{array}{l}\text { Mandibular } \\
\text { izquierdo }\end{array}$ & $\begin{array}{l}\text { Extracción } \\
\text { dental }\end{array}$ \\
\hline$N^{\circ} 8$ & Femenino & 78 & $\begin{array}{l}\text { Cáncer de } \\
\text { mama }\end{array}$ & $\begin{array}{c}\text { Ácido } \\
\text { zolendrónico }\end{array}$ & 20 meses & 2 & $\begin{array}{l}\text { Mandibular } \\
\text { derecho }\end{array}$ & $\begin{array}{l}\text { Extracción } \\
\text { dental }\end{array}$ \\
\hline$N^{\circ} 9$ & Femenino & 51 & $\begin{array}{c}\text { Cáncer de } \\
\text { mama }\end{array}$ & $\begin{array}{c}\text { Ácido } \\
\text { zolendrónico }\end{array}$ & 38 meses & 3 & $\begin{array}{l}\text { Mandibular } \\
\text { izquierdo }\end{array}$ & $\begin{array}{l}\text { Extracción } \\
\text { dental }\end{array}$ \\
\hline
\end{tabular}




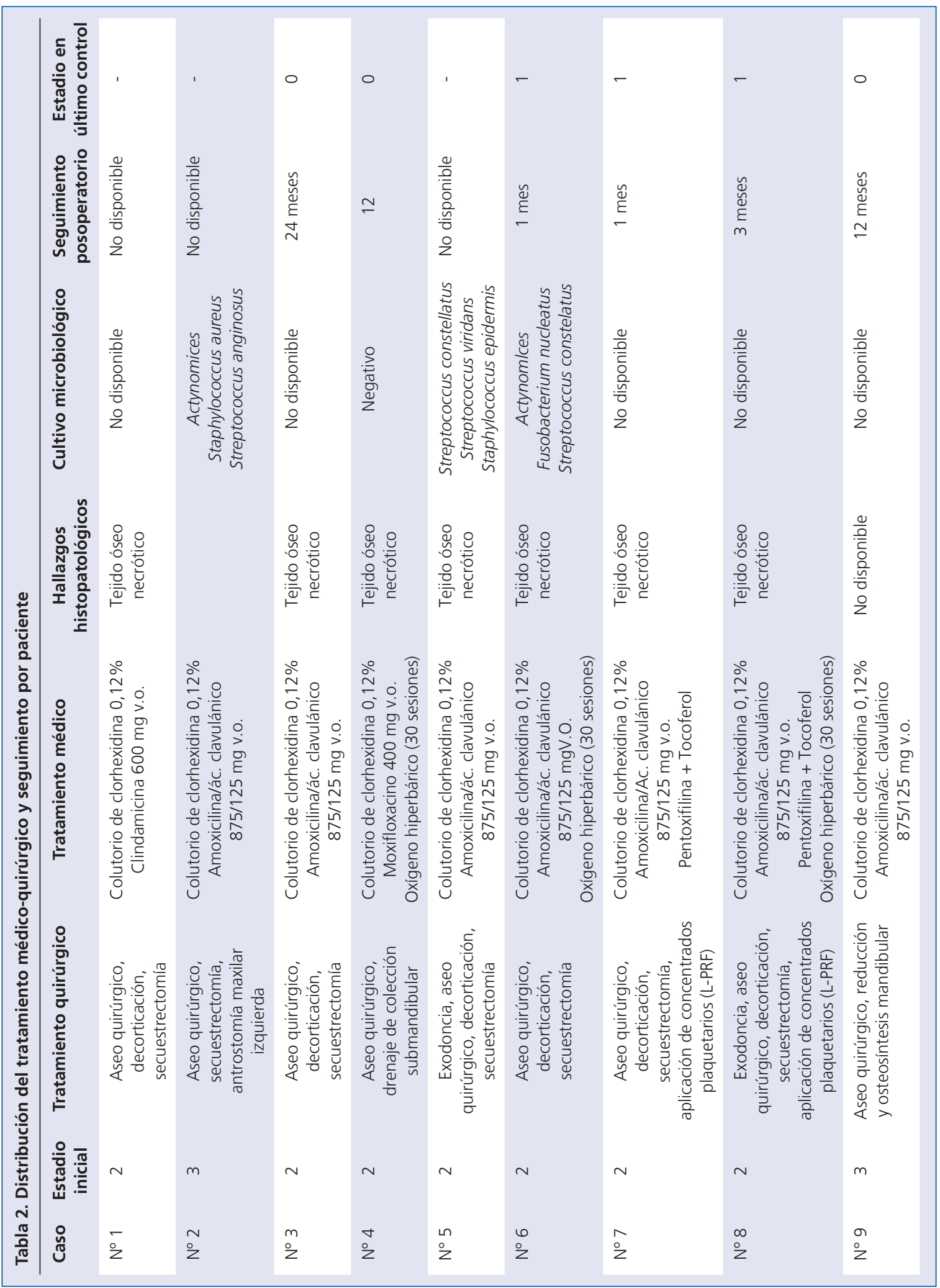


caso se efectuó además tratamiento con rituximab. Los diagnósticos de base que motivaron la indicación de terapia antirresortiva fueron: cáncer de mama con metástasis óseas $(\mathrm{N}=5)$, mieloma múltiple $(\mathrm{N}=2)$ y osteoporosis $(\mathrm{N}=2)$. El bifosfonato más frecuente en esta serie de casos fue el ácido zoledrónico. El tiempo promedio de tratamiento con BFIV, previo al diagnóstico de la OMAM fue de 37,5 meses.

De los 9 pacientes estudiados, en 8 se realizó exodoncias en la zona donde se desarrolló la OMAM. En un caso la etiología se atribuyó a sobrecompresión protésica, ninguno desarrolló osteonecrosis de manera espontánea. De 9 casos, 7/9 de las OMAM se ubicaron en la mandíbula y $2 / 9$ en el maxilar.

De los 9 pacientes, 7 presentaron hueso necrótico expuesto asociado a dolor local (estadio II) al momento del diagnóstico. Por otro lado, 2/9 de pacientes se presentaron con estadios avanzados de la enfermedad (III) al momento del examen inicial, manifestándose como necrosis de reborde alveolar extendido al seno maxilar y fractura patológica de ángulo mandibular.

La biopsia del tejido óseo necrótico fue realizada en 8/9 casos. Dentro de los hallazgos histopatológicos se describió hueso parcial o completamente necrótico, con distintos grados de infiltrado inflamatorio y colonias bacterianas. Fueron enviadas muestras para cultivo microbiológico en 4 casos. Los tipos bacterianos descritos incluyen Actynomices, Staphylococcus aureus, Streptococcus anginosus, Streptococcus constellatus, Streptococcus grupo viridans, Staphylococcus epidermidis y Fusobacterium nucleatum. El tratamiento quirúrgico en la mayoría de los casos consistió en aseo quirúrgico, decorticación y secuestrectomía; sin embargo, en los casos diagnosticados en estadio III fue necesario realizar procedimientos más invasivos (Tabla 2).

En uno de ellos se realizó una hemimaxilectomía parcial por compromiso del seno maxilar. En el segundo caso, la paciente se presentó con una fractura patológica del ángulo mandibular, por lo que fue necesario realizar una reducción y fijación con elementos de osteosíntesis. En dos casos se utilizaron concentrados plaquetarios (fibrina rica en plaquetas o L-PRF) aplicados en forma de membrana bajo el colgajo mucoperióstico.
Todos los pacientes recibieron en el posoperatorio colutorios de clorhexidina al $0,12 \%$ cada 8 a 12 horas, y tratamiento antibiótico sistémico (Tabla 2). En 2 casos fue indicado como terapia complementaria la administración de pentoxifilina + tocoferol (PENT-E). Tres pacientes recibieron al menos un ciclo de 30 sesiones de oxígeno hiperbárico.

\section{Discusión}

La OMAM corresponde a una complicación secundaria al tratamiento con fármacos antirresortivos o antiangiogénicos. Los fármacos antirresortivos se definen como aquellos que afectan el recambio óseo y están indicados en el manejo de enfermedades con aumento de la resorción ósea, como la hipercalcemia maligna, mieloma múltiple y metástasis óseas de tumores sólidos ${ }^{1-9}$. Fármacos como los bifosfonatos y denosumab se incluyen en este grupo de medicamentos.

Los bifosfonatos actúan a nivel óseo en los sitios de metabolismo activo, inhibiendo la actividad de los osteoclastos y aumentando su grado de apoptosis ${ }^{11-13}$. Esto se traduce en una inhibición de la resorción ósea, disminución en el recambio óseo y un aumento en su densidad, con efectos prolongados y persistentes en el tiempo ${ }^{12-14}$. Los bifosfonatos administrados vía intravenosa (BFIV), como el ácido zoledrónico y el pamidronato, se utilizan habitualmente como parte del tratamiento de la osteoporosis severa, metástasis óseas e hipercalcemia maligna. Por otra parte, los bifosfonatos administrados por vía oral (BFO) se indican principalmente para el tratamiento de osteoporosis ${ }^{15}$. Denosumab corresponde a un anticuerpo monoclonal que actúa sobre los receptores activadores del factor nuclear Kappa $B$ ligando (RANK-L), un componente clave en la formación y activación de los osteoclastos ${ }^{2,11}$.

Respecto a fármacos antiangiogénicos, estos inhiben la formación de vasos sanguíneos, interrumpiendo su cascada de señalización. Están indicados en el manejo de tumores gastrointestinales, carcinomas renales, tumores neuroendocrinos y otras lesiones malignas ${ }^{2}$. Sunitinib o bevacizumab son ejemplos de estos $^{1}$. Sunitinib inhibe las señales celulares por unión a múltiples receptores de tirosina 
quinasa, incluyendo los receptores de factor de crecimiento derivado de plaquetas y del factor de crecimiento endotelial, los que que actúan en la angiogénesis y la proliferación de células tumorales. Por su parte el bevacizumab corresponde a un anticuerpo monoclonal que se une al factor de crecimiento endotelial vascular, inhibiendo la señalización de la angiogénesis ${ }^{1-2}$.

Si bien los reportes de OMAM han aumentado desde el año $2003^{1}$, la real incidencia se desconoce con exactitud ${ }^{16-18}$. Las tasas de prevalencia varían entre un $0,94 \%$ a $13 \%$ en diferentes grupos de población con distintos esquemas farmacológicos, siendo el mayor factor desencadenante, la exodoncia u otros procedimientos odontológicos invasivos ${ }^{19-21}$. En Red de Salud UC-Christus la prevalencia de OMAM en pacientes tratados con BFIV es de 1,4\% (143 pacientes analizados), en donde el $78 \%$ de los casos presentaban patologías de origen oncológico, como cáncer de próstata, cáncer de mama y mieloma múltiple ${ }^{22}$.

En 8/9 de los casos con OMAM se asoció a la exodoncia como factor odontológico desencadenante. De estos, en solo uno de ellos la exodoncia se llevó a cabo en el mismo centro donde se realizó el estudio, como parte de la eliminación de focos odontogénicos previo al inicio de quimioterapia. En los 7 casos restantes la extracción dental fue realizada en otro centro, por lo cual no fue posible evaluar el diagnóstico de los dientes extraídos previamente o la presencia de focos infecciosos agudos que hayan podido participar como desencadenantes de la necrosis ósea.

Todos los pacientes de esta serie corresponden al género femenino, lo cual puede explicarse debido a que patologías de mayor prevalencia como el cáncer de mama y la osteoporosis afectan en mayor medida a mujeres. Sin embargo, pacientes oncológicos de género masculino en tratamiento con antirresortivos son igual de propensos a desarrollar OMAM. En todos los casos, los pacientes tenían historia de tratamiento con BFIV. Estos resultados son concordantes con Bagán et al. (2018), quienes reportaron 183 pacientes con OMAM, observando que el $30,1 \%$ de los casos tuvo cáncer de mama como diagnóstico primario, y 72,7\% de los pacientes habrían sido tratados con BFIV ${ }^{21}$.

Respecto al tiempo transcurrido desde el inicio del tratamiento con bifosfonatos y el desarrollo de la OMAM, se ha descrito que este podría variar entre 3 a 9 años dependiendo el tipo de bifosfonatos y la vía de administración ${ }^{1,22}$. En nuestra serie, el paciente en tratamiento con alendronato fue diagnosticado con OMAM 5 años después de iniciado su tratamiento antirresortivo, mientras que en pacientes tratados con ácido zoledrónico el tiempo promedio fue 3,16 años meses desde la primera dosis. Independientemente de lo anterior, es posible que el desarrollo de una OMAM esté más relacionado temporalmente con un evento desencadenante en específico (por ejemplo, una exodoncia y/o una infección odontogénica) que con la duración de la terapia farmacológica. También es posible suponer que el tiempo de aparición de los primeros síntomas es menor al reportado, ya que, en este grupo en particular de pacientes estudiados, el diagnóstico se realizó en estadios avanzados de la enfermedad. Por lo tanto, es fundamental el diagnóstico precoz de patologías odontológicas que requieran de tratamiento quirúrgico de piezas dentarias y hueso alveolar previo al inicio de terapia con BFIV.

En relación a la ubicación anatómica de OMAM, 7/9 casos se desarrollaron a nivel mandibular y en solo $2 / 9$ de los casos la necrosis comprometió el maxilar. Esto es similar a lo reportado en la literatura, donde el compromiso maxilar se describe entre el 25,7\%-36,5\% de los $\operatorname{casos}^{6,21,23}$. La mayor frecuencia de aparición de la OMAM a nivel mandibular ha sido atribuida al menor grado de vascularización y a una mayor proporción de tejido óseo corticalizado en comparación al maxilar ${ }^{1-2}$.

El tratamiento de la OMAM varía dependiendo del estadio de la enfermedad. Si bien no existe consenso al respecto, basados en los datos disponibles en la literatura y el trabajo interdisciplinario de los equipos de cirugía oncológica y maxilofacial, y oncología médica (Red de Salud UC-Christus), se propone el siguiente protocolo para la prevención y manejo, según estadio de la $\mathrm{OMAM}^{10}$ :

\section{- Estadios 0 y I}

- Proporcionar tratamiento sintomático y manejar factores locales.

- Uso de antisépticos tópicos. 
- Antibioterapia sistémica, en presencia de signos de infección.

- Pentoxifilina $400 \mathrm{mg}+$ tocoferol $400 \mathrm{UI}$ (PENTE-E) cada 12 horas, hasta resolución de los síntomas.

\section{- Estadios II y III}

- Tratamiento médico-quirúrgico.

- Uso de antisépticos tópicos.

- Antibioterapia sistémica (amoxicilina/ ácido clavulánico 875/125 mg c/12 horas) por 7-14 días.

- Pentoxifilina $400 \mathrm{mg}+$ tocoferol $400 \mathrm{UI}$ (PENTE-E) cada 12 horas, hasta evidencia clínica e imagenológica de resolución del cuadro.

- Aseo quirúrgico, decorticación y resección de secuestros óseos.

- Cierre por primera intención, colgajos libres de tensión y uso adicional de concentrados plaquetarios (L-PRF).

- Biopsia diferida de secuestros óseos.

- Cultivos de aerobios y anaerobios.

- Valorar el aporte de suspensión de la terapia antirresortiva en cada caso.

En nuestro estudio, el tratamiento quirúrgico se vio asociado a una disminución de los síntomas, en conjunto con una disminución de los estadios clínicos. El tratamiento quirúrgico de la OMAM en estadios II y III tiene por objetivo eliminar el tejido óseo necrótico, realizar un aseo quirúrgico y eliminar tejido infeccioso. Para mejorar el pronóstico del procedimiento quirúrgico, es importante el cierre primario de la exposición ósea. López-Jornet y cols. (2016) reportó que la resección quirúrgica de los secuestros óseos en conjunto con tratamiento antibiótico con amoxicilina y ácido clavulánico, asociado al uso de colutorios de clorhexidina no logra una curación completa de la enfermedad en todos los casos; sin embargo, genera una mejora significativa de los síntomas en más del $80 \%$ de los casos de OMAM en estadio II y III ${ }^{23}$.

En términos de evaluación clínica de éxito terapéutico, este se ha definido como: 1) ausencia de exposiciones óseas, 2) salud de las mucosas y 3 ) disminución del estadio de OMAM. Estas en conjunto serían un buen indicador para definir estrategias terapéuti- cas a seguir. Vanpoecke y cols. describió que bajo estos parámetros, los estadios II y III no tendrían mejoras clínicas con estrategias no invasivas de tratamiento, haciendo énfasis en que para estos casos el tratamiento quirúrgico agresivo, en conjunto con reconstrucciones microvascularizadas para estadios III, tendría tasas de éxito del 97\% ${ }^{24}$.

En relación con los estadios II, Zirk y cols. destacan el rol de la cirugía resectiva agresiva, donde los cultivos y antibiogramas cobran vital importancia para limitar el progreso de la enfermedad a un estadio superior y por consiguiente disminuir la comorbilidad que esto conlleva ${ }^{25}$. Del total de pacientes estadio II, el $95,1 \%$ no requirió de resecciones segmentarias gracias al diagnóstico precoz, decorticaciones agresivas y uso de antibioterapia sistémica. En relación con el estudio bacteriológico, se observó anaerobios facultativos y anaerobios estrictos en un $67,3 \%$, con una susceptibilidad a la ampicilina/sulbactam de $79,1 \%{ }^{25}$. En los casos presentados en este estudio, se observó la presencia de cultivos similares, conformados principalmente por Actynomices, Streptococcus y Fusobacterium, los cuales no presentaron resistencia al tratamiento con amoxicilina y ácido clavulánico. Se presentaron dos casos con alergias a penicilinas, por lo que se decidió el tratamiento vìa oral con moxifloxacino o clindamicina clorhidrato.

La definición de éxito quirúrgico como herramienta de toma decisiones no debe dejar de lado la calidad de vida del paciente. Muchos de ellos ya presentan un deterioro de su calidad de vida debido al diagnóstico oncológico de base, por lo que un enfoque interdisciplinario es crucial para los casos de OMAM iniciales o más avanzados.

Otro de los elementos que ha tomado mayor relevancia últimamente en el tratamiento de la OMAM es el uso de la combinación de pentoxifilina con tocoferol (PENT-E). Su aplicación fue descrita inicialmente para el tratamiento de la osteorradionecrosis y ha mostrado resultados prometedores tanto para la prevención como tratamiento de la $\mathrm{OMAM}^{26-29}$. La pentoxifilina tiene la capacidad de generar dilatación de vasos sanguíneos periféricos, mejorando la irrigación del tejido óseo comprometido. El tocoferol (vitamina E) es un potente antioxidante que disminuye la 
fibrosis tisular ${ }^{28,29}$. Esta terapia es bien tolerada, de bajo costo, y ha reportado resultados favorables en cuanto a la resolución de síntomas, cicatrización ósea y de mucosa oral en pacientes con OMAM. La recomendación es el uso de pentoxifilina $400 \mathrm{mg}$ y tocoferol $400 \mathrm{UI}, 3$ veces al día, hasta la resolución del cuadro de osteonecrosis ${ }^{26-29}$. Esta indicación se realizó en los casos más recientes, ya que previo al año 2014 no existía evidencia suficiente como para sugerir su uso ${ }^{26-29}$.

El uso de oxígeno hiperbárico (OHB) también ha sido descrito en la literatura como una alternativa de tratamiento de casos avanzados de OMAM, ya que tendría la capacidad de promover la angiogénesis y cicatrización ósea debido a la producción de especies reactivas de nitrógeno y oxígeno ${ }^{30}$. Lamentablemente, su impacto en el manejo de la OMAM aún es controversial. Un estudio clínico randomizado describió que, clínicamente, el OHB parece ser un complemento útil al tratamiento, ya que además de promover angiogénesis y formación ósea tendría un rol importante en el manejo del dolor y calidad de vida, en particular para los casos más graves refractarios a tratamiento habitual ${ }^{30,31}$. En nuestro estudio 3 pacientes recibieron al menos un ciclo de 30 sesiones de OHB como tratamiento complementario. En estos pacientes hubo disminución de sintomatología y del estadio clínico de su enfermedad. Sin embargo, no se puede determinar la real eficacia y utilidad del OHB con este estudio.

Dentro del tratamiento médico-quirúrgico, un complemento de utilidad para favorecer la cicatrización del tejido óseo y de la mucosa en pacientes con OMAM es el uso de concentrados plaquetarios autólogos, como plasma rico en plaquetas (PRP) o fibrina rica en plaquetas $(\mathrm{PRF})^{32}$. Estos concentrados aportan factores de crecimiento que estimulan la angiogénesis y la diferenciación celular, además de tener efectos antiinflamatorios. López-Jornet y cols. reportaron que el uso de estos concentrados plaquetarios en conjunto con el tratamiento quirúrgico, logran una resolución completa en un $85,98 \%$ de los casos ${ }^{32}$. La obtención de concentrados plaquetarios es un procedimiento simple y de bajo costo, por lo que en los últimos años hemos adoptado este procedimiento como parte del tratamiento médico-quirúrgico de estos pacientes.
Los pacientes reportados en esta serie de casos repararon en su salud oral o consultaron a un especialista cuando la lesión ya se encontraba en etapas avanzadas. Lamentablemente, el manejo integral de las complicaciones buco-maxilofaciales de los pacientes con OMAM, especialmente el tratamiento quirúrgico, puede interferir con el tratamiento de las patologías de base que motivaron la terapia antirresortiva. Es por esto que se hace énfasis en que los pacientes que requieren iniciar tratamiento con antirresortivos o antiangiogénicos, deben ser sometidos a una evaluación oral y maxilofacial centrada en el diagnóstico y la prevención de patologías que puedan requerir terapia odontológica invasiva, resolviendo todo foco infeccioso real o potencial, antes de iniciados los antirresortivos y/o antiangiogénicos.

\section{Conclusión}

A pesar del número de pacientes de nuestra revisión, podemos sugerir que el tratamiento médico-quirúrgico en estadios II y III de OMAM parece favorecer la disminución de los síntomas y el control de la infección buco-maxilofacial, teniendo como principio la eliminación de los secuestros óseos y el cierre primario de los tejidos. El uso de PENT-E y PRF como terapias coadyuvantes al tratamiento médico-quirúrgico pareciera favorecer el éxito terapéutico. La indicación de OHB se encontraría limitada a casos refractarios al tratamiento médico-quirúrgico, con el fin de alivio de síntomas y optimizar la calidad de vida.

Es fundamental la instauración de protocolos preventivos en este grupo de pacientes, que contemple la evaluación previa al inicio de terapias antirresortivas, y seguimiento clínico para evitar una evolución a estadios avanzados de OMAM. Se requiere contar con más trabajos prospectivos que permitan una mayor comprensión y optimización del tratamiento de esta patología.

\section{Bibliografía}

1. Marx RE. Pamidronate (Aredia) and zoledronate (Zometa) induced avascular necrosis of the jaws: a growing epidemic. J Oral Maxillofac 
Surg. 2003;61(9):1115-1117. doi: 10.1016/s02782391(03)00720-1.

2. Ruggiero SL, Dodson TB, Fantasia J, et al. American Association of Oral and Maxillofacial Surgeons position paper on medication-related osteonecrosis of the jaw--2014 update. J Oral Maxillofac Surg. 2014;72(10):1938-1956. doi: 10.1016/j. joms.2014.04.031.

3. Khan AA, Morrison A, Hanley DA, et al. Diagnosis and management of osteonecrosis of the jaw: a systematic review and international consensus. J Bone Miner Res. 2015;30(1):3-23. doi: 10.1002/ jbmr.2405.

4. Hellstein JW, Adler RA, Edwards B, et al. Managing the care of patients receiving antiresorptive therapy for prevention and treatment of osteoporosis: executive summary of recommendations from the American Dental Association Council on Scientific Affairs. J Am Dent Assoc. 2011;142(11):1243-1251. doi: 10.14219/jada.archive.2011.0108.

5. King AE, Umland EM. Osteonecrosis of the jaw in patients receiving intravenous or oral bisphosphonates. Pharmacotherapy. 2008;28(5):667677. doi: 10.1592/phco.28.5.667.

6. Mavrokokki T, Cheng A, Stein B, Goss A. Nature and frequency of bisphosphonate-associated osteonecrosis of the jaws in Australia. J Oral Maxillofac Surg. 2007;65(3):415-423. doi: 10.1016/j.joms.2006.10.061.

7. Woo SB, Hellstein JW, Kalmar JR. Narrative review: bisphosphonates and osteonecrosis of the jaws. Ann Intern Med. 2006;144(10):753-761. doi: 10.7326/0003-4819-144-10-200605160-00009.

8. Bagán J, Blade J, Cozar JM, et al. Recommendations for the prevention, diagnosis, and treatment of osteonecrosis of the jaw (ONJ) in cancer patients treated with bisphosphonates. Med Oral Patol Oral Cir Bucal. 2007;12(4):E336-E340.

9. Yamashita J, McCauley LK, Van Poznak C. Updates on osteonecrosis of the jaw. Curr Opin Support Palliat Care. 2010;4(3):200-206. doi: 10.1097/ SPC.0b013e32833d303b.

10. Foncea C, von Bischhoffshausen K, Teuber C, y cols. Osteonecrosis de los maxilares asociada a medicamentos: revisión de la literatura y propuesta para la prevención y manejo [Osteonecrosis of the jaws]. Rev Med Chil. 2020;148(7):983-991. doi: 10.4067/S0034-98872020000700983.

11. Hess LM, Jeter JM, Benham-Hutchins M, Alberts DS. Factors associated with osteonecrosis of the jaw among bisphosphonate users. Am J Med. 2008;121(6):475-483.e3. doi: 10.1016/j. amjmed.2008.01.047.

12. Nancollas GH, Tang R, Phipps RJ, y cols. Novel insights into actions of bisphosphonates on bone: differences in interactions with hydroxyapatite. Bone. 2006;38(5):617-627. doi: 10.1016/j.bone.2005.05.003.

13. McClung M, Harris ST, Miller PD, y cols.
Bisphosphonate therapy for osteoporosis: benefits, risks, and drug holiday. Am J Med. 2013;126(1):13-20. doi: 10.1016/j.amjmed.2012.06.023.

14. Russell RG. Bisphosphonates: mode of action and pharmacology. Pediatrics. 2007;119 Suppl 2:S150-S162. doi: 10.1542/peds.2006-2023H

15. Sawatari Y, Marx RE. Bisphosphonates and bisphosphonate induced osteonecrosis. Oral Maxillofac Surg Clin North Am. 2007;19(4):487-vi. doi: 10.1016/j.coms.2007.07.003

16. McLeod NM, Brennan PA, Ruggiero SL. Bisphosphonate osteonecrosis of the jaw: a historical and contemporary review. Surgeon. 2012;10(1):36-42. doi: 10.1016/j.surge.2011.09.002.

17. Otto S, Pautke C, Van den Wyngaert T, Niepel D, Schiødt M. Medication-related osteonecrosis of the jaw: Prevention, diagnosis and management in patients with cancer and bone metastases. Cancer Treat Rev. 2018;69:177-187. doi: 10.1016/j. ctrv.2018.06.007.

18. Otto S. Antiresorptive drug-related osteonecrosis of the jaw (ARONJ)-a guide to research. 2016. K. E. Fleisher, \& R. Kontio (Eds.). Thieme.

19. Yarom N, Yahalom R, Shoshani Y, Hamed W, Regev E, Elad S. Osteonecrosis of the jaw induced by orally administered bisphosphonates: incidence, clinical features, predisposing factors and treatment outcome. Osteoporos Int. 2007;18(10):1363-1370. doi: 10.1007/ s00198-007-0384-2.

20. Bagan L, Jiménez Y, Leopoldo M, Murillo-Cortes J, Bagan J. Exposed necrotic bone in 183 patients with bisphosphonate-related osteonecrosis of the jaw: Associated clinical characteristics. Med Oral Patol Oral Cir Bucal. 2017;22(5):e582-e585. Published 2017 Sep 1. doi: 10.4317/medoral.2213.

21. Teuber LC, Foncea RC Rojas CF, von Bischhoffshausen PK, Goñi EI, Vargas DÁ, et al. Prevalencia de osteonecrosis de los maxilares asociada a medicamentos en pacientes tratados con bifosfonatos intravenosos: análisis epidemiológico en Centro del Cáncer - Red de Salud UCCHRISTUS. Rev Otorrinolaringol Cir Cabeza Cuello. 2020; 80(4):469-476. doi: 10.4067/S071848162020000400469 .

22. Aljohani S, Gaudin R, Weiser J, et al. Osteonecrosis of the jaw in patients treated with denosumab: A multicenter case series. J Craniomaxillofac Surg. 2018;46(9):1515-1525. doi: 10.1016/j. jcms.2018.05.046.

23. Klingelhöffer C, Zeman F, Meier J, Reichert TE, Ettl T. Evaluation of surgical outcome and influencing risk factors in patients with medication-related osteonecrosis of the jaws. J Craniomaxillofac Surg. 2016;44(10):1694-1699. doi: 10.1016/j. jcms.2016.08.001.

24. Vanpoecke J, Verstraete L, Smeets M, Ferri J, Nicot R, Politis C. Medication-related osteonecrosis of the jaw 
(MRONJ) stage III: Conservative and conservative surgical approaches versus an aggressive surgical intervention: A systematic review. J Craniomaxillofac Surg. 2020;48(4):435-443. doi: 10.1016/j. jcms.2020.02.017.

25. Zirk M, Kreppel M, Buller J, et al. The impact of surgical intervention and antibiotics on MRONJ stage II and III - Retrospective study. J Craniomaxillofac Surg. 2017;45(8):1183-1189. doi: 10.1016/j. jcms.2017.05.027.

26. Delanian S, Chatel C, Porcher R, Depondt J, Lefaix JL. Complete restoration of refractory mandibular osteoradionecrosis by prolonged treatment with a pentoxifylline-tocopherol-clodronate combination (PENTOCLO): a phase II trial. Int J Radiat Oncol Biol Phys. 2011;80(3):832-839. doi: 10.1016/j. ijrobp.2010.03.029.

27. Patel V, Gadiwalla Y, Sassoon I, Sproat C, Kwok J, McGurk M. Prophylactic use of pentoxifylline and tocopherol in patients who require dental extractions after radiotherapy for cancer of the head and neck. Br J Oral Maxillofac Surg. 2016;54(5):547-550. doi: 10.1016/j.bjoms.2016.02.024.

28. Magremanne M, Reychler H. Pentoxifylline and tocopherol in the treatment of yearly zoledronic acidrelated osteonecrosis of the jaw in a corticosteroid- induced osteoporosis. J Oral Maxillofac Surg. 2014;72(2):334-337. doi: 10.1016/j.joms.2013.06.188.

29. Yalcin-Ülker GM, Cumbul A, Duygu-Capar G, Uslu Ü, Sencift K. The role of pentoxifylline on the medication-related osteonecrosis of the jaw via expression of vascular endothelial growth factor in a rat model. International Journal of Oral and Maxillofacial Surgery. 2017;46, 151. doi: 10.1016/j. ijom.2017.02.522.

30. Al Hadi H, Smerdon GR, Fox SW. Hyperbaric oxygen therapy accelerates osteoblast differentiation and promotes bone formation. J Dent. 2015;43(3):382388. doi: 10.1016/j.jdent.2014.10.006.

31. Freiberger JJ, Padilla-Burgos R, McGraw T, et al. What is the role of hyperbaric oxygen in the management of bisphosphonate-related osteonecrosis of the jaw: a randomized controlled trial of hyperbaric oxygen as an adjunct to surgery and antibiotics. J Oral Maxillofac Surg. 2012;70(7):1573-1583. doi: 10.1016/j. joms.2012.04.001.

32. Lopez-Jornet P, Sanchez Perez A, Amaral Mendes $\mathrm{R}$, Tobias A. Medication-related osteonecrosis of the jaw: Is autologous platelet concentrate application effective for prevention and treatment? A systematic review. J Craniomaxillofac Surg. 2016;44(8):10671072. doi: 10.1016/j.jcms.2016.05.004. 